Original Article

\title{
Acceptance of COVID-19 vaccination at different hypothetical efficacy and safety levels in ten countries in Asia, Africa, and South America
}

\begin{abstract}
Dott F. Rosiello', Samsul Anwar², Amanda Yufika3, Rashed Y. Adam4, Mohajer IH. Ismaeil5, Asma Y. Ismail6, Nesrine BH. Dahman7, Montacer Hafsi7, Manel Ferjani7, Farah S. Sami, Fatma A Monib9, Subramaniam R'10, Sunil Anandu'1, Md Ariful Haque12, Lirane ED. Ferreto $^{13}$, José TO. Aburto'14, Jorge ET. Rojas14, Seyi S. Enitan'5, Akele R. Yomi' ${ }^{16}$, Eyiuche D. Ezigbo17, Elham Babadi18, Edris Kakemam ${ }^{19}$, Najma I. Malik2o, Irfan Ullah²1 and Malik Sallam²,23,24*

${ }^{1}$ Department of Public Health and Infectious Disease, Sapienza-University of Rome, Rome, Italy; ${ }^{2}$ Department of Statistics, Faculty of Mathematics and Natural Sciences, Universitas Syiah Kuala, Banda Aceh, Indonesia; 3 Department of Family Medicine, School of Medicine, Universitas Syiah Kuala, Banda Aceh, Indonesia; 4Omdurman Teaching Hospital, Khartoum, Sudan; 5Faculty of Medicine, Alzaiem Alazhari University, Khartoum, Sudan; ${ }^{6}$ Department of Internal Medicine, Faculty of Medicine, Sudan International University, Khartoum, Sudan; 7 Faculty of Medicine of Tunis, University of Tunis El Manar, Tunisia; ${ }^{8}$ Faculty of Medicine, Ain Shams University, Nasr City, Cairo, Egypt; 9Faculty of Medicine, Assiut University, Assiut, Egypt; ${ }^{10}$ Department of Public Health Dentistry, Indira Gandhi Institute of Dental Sciences, Nellikuzhi, Kothamangalam, Kerala, India; ${ }^{11}$ Division of Veterinary Parasitology, ICAR-Indian Veterinary Research Institute, Izatnagar, Bareilly, Uttar Pradesh, India; ${ }^{12}$ Department of Orthopedic Surgery, Yan'an Hospital Affiliated to Kunming Medical University, Kunming, Yunnan, China; ${ }^{13}$ Department of Public Health and Postgraduate Program in Applied Health Sciences, Faculty of Medicine, Western Paraná State University, Brazil; ${ }^{14}$ Faculty of Medicine, University of La Frontera, Temuco, Chile; ${ }^{15}$ Department of Medical Laboratory Science, Babcock University, Ilishan-Remo, Ogun State, Nigeria; ${ }^{16}$ Department of Medical Laboratory Science, Afe Babalola University, Ado Ekiti, Nigeria; ${ }^{17}$ Department of Medical Laboratory Sciences, Faculty of Health Sciences \& Technology University of Nigeria, Enugu State Nigeria; ${ }^{18}$ Research Fellow, Mayo Clinic, Rochester, USA; 19 Iranian Center of Excellence in Health Management, School of Management and Medical Informatics, Tabriz University of Medical Sciences, Tabriz, Iran; ${ }^{20}$ Department of Psychology, University of Sargodha, Sargodha, Pakistan; ${ }^{21}$ Department of Internal Medicine, Kabir Medical College, Gandhara University, Peshawar, Pakistan; ${ }^{22}$ Department of Pathology, Microbiology and Forensic Medicine, School of Medicine, The University of Jordan, Amman Jordan; ${ }^{23}$ Department of Clinical Laboratories and Forensic Medicine, Jordan University Hospital, Amman Jordan; ${ }^{24 D e p a r t m e n t ~ o f ~ T r a n s l a t i o n a l ~ M e d i c i n e, ~ F a c u l t y ~ o f ~}$
\end{abstract} Medicine, Lund University, Malmö, Sweden

*Corresponding author: malik.sallam@ju.edu.jo

\section{Abstract}

Vaccine hesitancy, defined as the reluctance or rejection in receiving a vaccine despite its availability, represents a major challenge to global health efforts aiming to control the ongoing COVID-19 pandemic. Understanding the possible factors correlated with COVID-19 vaccine hesitancy using a refined well-informed approach can be helpful to address the phenomenon. The current study aimed to evaluate COVID-19 vaccine acceptance rates using four hypothetical scenarios of varying levels of vaccine efficacy and safety profiles in ten Asian, African and South American countries. These scenarios included: 95\% efficacy and 20\% side effects (Vaccine A), 75\% efficacy and 5\% side effects (Vaccine B); 75\% efficacy and 20\% side effects (Vaccine C) and 50\% efficacy and 5\% side effects (Vaccine D). This study used a self-administered online survey that was distributed during February-May 2021. The total number of study respondents was 1337 with countries of residence as follows: India (21.1\%), Pakistan (12.9\%), Sudan (11.2\%), Nigeria (9.3\%), Iran (8.2\%), Bangladesh and Brazil (7.9\%), Chile (7.7\%), Tunisia (7.6\%), and Egypt (6.2\%). The overall acceptance rates for COVID-19 vaccination were variable based on varying degrees of safety and efficacy as follows: $55.6 \%$ for Vaccine C, $58.3 \%$ for Vaccine D, 74.0\% for Vaccine A and 80.1\% for Vaccine B. The highest levels of COVID-19 
vaccine acceptance were observed in Brazil followed by Chile across the four different safety and efficacy scenarios. The lowest COVID-19 vaccine acceptance rates were reported in Egypt and Tunisia for the low safety scenarios (20\% side effects), and the low efficacy scenario (50\% efficacy). The study revealed the potential effect of vaccine safety and efficacy on the intention to get COVID-19 vaccination. At the same efficacy level, higher possibility of side effects caused a large drop in COVID-19 vaccine acceptance rate. This indicates the importance of accurate communication regarding vaccine safety and efficacy on attitude towards the vaccine and intentions to get vaccinated. Regional differences in COVID-19 vaccine acceptance were observed with the Middle East/North African countries showing the lowest rates and the South American countries displaying the highest vaccine acceptance rates.

Keywords: COVID-19, COVID-19 vaccine, acceptance, hesitancy, WHO SAGE

\section{Introduction}

$T$

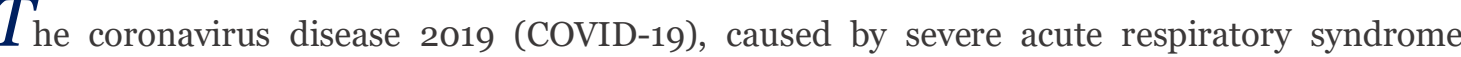
coronavirus 2 (SARS-CoV-2), has been a major global health threat since first being declared as a pandemic by the World Health Organization (WHO) in March 2020 [1, 2]. The mitigation strategies and personal protective measures, such as movement restrictions, social distancing, and face mask-wearing practices, have been implemented in various countries regarding COVID-19 prevention [3]. Although such efforts have helped to minimize the spread of SARSCoV-2 infection and contributed to the epidemic curve flattening, the pandemic is still ongoing [4]. The COVID-19 resurgence has been reported coinciding with societies, businesses, and economies reopening $[5,6]$, suggesting the urgent need for long-term preventive measures. Vaccines could be implemented as a long-term protective measure in controlling COVID-19 [7].

Vaccination is a fundamental strategy for controlling and preventing infectious diseases [8, 9]. Given a high rate of COVID-19-associated morbidity and mortality, various COVID-19 vaccines have been developed and manufactured [10,11]. The first vaccine was developed in March 2020 by Moderna [10], and as of October 2021, approximately 132 vaccines have undergone clinical trials on humans; 13 vaccines in limited use; and 8 vaccines have been permitted for full use at least in one country [12]. Despite the initiation of COVID-19 vaccination since the beginning of 2021 [13], the total vaccine uptake globally, as of October 2021 was still less than 50\% [14]. Low COVID-19 vaccine coverage is multifactorial [15]; however, vaccine hesitancy and acceptance significantly affected vaccine decision and vaccination drive [16-19]. Vaccine hesitancy, by the World Health Organization Strategic Advisory Group of Experts (WHO SAGE), is defined as a "delay in acceptance or refusal of vaccination despite the ability of vaccination services" [20].

Previous studies have demonstrated high heterogeneity in the willingness to accept COVID-19 vaccines among different countries worldwide [17, 19]. Various sociodemographic factors, including race, age, educational level, occupation and income; and attitudes and beliefs in COVID-19 infection; trust in central government; mistrust or faltering in vaccines safeness; employers' mandate or recommendation; belief in conspiracy theories; vaccine effectiveness; as well as vaccine-associated misinformation spread have significantly contributed to COVID-19 vaccine acceptance or hesitancy [17, 21-26]. Many investigations have been performed regarding the acceptance of COVID-19 vaccines among societies in high-income countries (HICs) [22, 25, 27-29]; however, similar studies in countries (LMICs) were still limited and did not represent all LMICs [19]. In addition, vaccine distribution in HICs is relatively high, while in LMICs, the distribution has been reportedly low [30]. Given the fact that vaccine uptake in some LMICs, such as Sudan, Iraq, India is still low (<20\%) [13], research regarding vaccine acceptance among populations in these countries is highly important.

The differences in vaccine acceptance, causing differences in vaccine coverage among countries, could potentially lead to the delay of the global control of the pandemic, ensuing in the delay of societal and economic recovery [17]. This is particularly important considering the continuous emergence of SARS-CoV-2 lineages with potential vaccine escape properties [31]. 
Hence, determining the acceptance of COVID-19 vaccines within the community during the pandemic is highly warranted. Understanding and addressing factors associated with the willingness to accept a COVID-19 vaccine are crucial as they will help in formulating the best approach or action to accelerate the global vaccination rate and increase vaccine coverage to meet the requirements for population immunity worldwide [32]. The aim of this study was to assess the acceptance of COVID-19 vaccines among communities in ten LMICs in Asia, Africa, and South America during the COVID-19 pandemic, as well as to explore determinants of vaccination.

\section{Methods}

\section{Study setting and instrument}

Between February to May 2021, an online self-administered survey was conducted in four Asian countries (Bangladesh, India, Iran, and Pakistan), four African countries (Egypt, Nigeria, Sudan, and Tunisia), and two countries in South America (Brazil and Chile). All the countries are classified as low- or middle-income countries. The survey was hosted by SurveyMonkey and to recruit the participants, the invitations to participate in the survey were distributed on three social media and instant messaging platforms: Facebook, Twitter and WhatsApp. The survey consisted of several sections. The first introductory section consisted of information about the study and an informed consent page. Only those who agreed to participate could open the next sections. The following sections comprised questions collecting information on demographic characteristics, current health condition, perceived risk towards COVID-19, economic disruption during COVID-19 pandemic, vaccine hesitancy, and attitudes towards social distancing. Some of the questions were adopted from previous studies [17, 21, 33]. It required approximately 10-15 minutes to complete the survey.

\section{Response variables}

The response variable of the study was acceptance of the hypothetical COVID-19 vaccines with different efficacy and safety levels. To assess the COVID-19 vaccine acceptance, the respondents were provided with a scenario: "Imagine that a new COVID-19 vaccine has just been developed. It has received the same testing as the adult influenza vaccine. The government is offering it as a free and optional vaccine." To assess the effect of vaccine properties, they were asked whether they would accept a COVID-19 vaccine if the vaccine is [95\%, $75 \%$ or $50 \%$ ] effective, with a [5\% or 20\%] chance of side effects like fever or local pain. There were four combinations of vaccine efficacies and the chances of the side effects provided: Vaccine A (95\% effective with a $20 \%$ chance of side effects); Vaccine B ( $75 \%$ effective with a $5 \%$ chance of side effects); Vaccine C ( $75 \%$ effective with a $20 \%$ chance of side effects); and Vaccine D (50\% effective with a $5 \%$ chance of side effects). For each combination, two possible answers were provided: "Yes" and "No" and no "Unsure" option was provided.

\section{Explanatory variables}

Some possible explanatory variables were collected and assessed. Demographic characteristics were collected and grouped for statistical proposes in the following manner: age (less than 20, 21-30, 31-40, 41-50 and >51 years old), gender, urbanicity (rural and urban), monthly household income ( $<$ US\$ 500, \$500-\$999, \$1,000-\$1,999, \$2,000-\$2,999, \$3,000-\$4,999, $\$ 5,000-\$ 7,999$ and $\geq \$ 8,000$ ), religion (Islam, Christian (Protestant/Methodist/Lutheran/ Baptist), Catholic, Hindu, and others (Mormon, Greek or Russian Orthodox, Jewish, and Buddhist), and types of job (healthcare-related sector and non- healthcare-related sector) and types of occupation (self-employed, employed for wages, out of work for less or more than one year, homemaker, student and retired or unable to work). Respondents were also asked whether they had received a flu vaccination in the past 12 months and whether they had comorbidities condition for COVID-19 such as hypertension, diabetes, cardiopulmonary diseases.

We also asked about the economic disruption experienced by respondents by asking two questions: "How much your work changed as a result of the COVID-19 pandemic?" and "How much your salary changed as a result of the COVID-19 pandemic?" The possible answers for the 
first question were: "I work fewer hours", "No change or not applicable (not working)", "I work more hours" and "I was let go from my job" while for the last question, three possible responses were: "I am getting paid less", "I am getting paid more" or "No change".

In addition, the attitude towards the benefit of vaccination and social distancing were assessed. To assess the vaccine hesitancy, six questions belong to lack of vaccination benefits construct from the WHO SAGE Vaccine Hesitancy Scale as used before [34] were used: (1) "Vaccines are important for my health"; (2) "All routine vaccines recommended by the healthcare workers are beneficial"; (3) "New vaccines carry more risks than older vaccines"; (4) "The information I receive about vaccines from the government is reliable and trustworthy; and (5) "Getting vaccines is a good way to protect me from disease". To assess the towards the benefits of social distancing, the respondents were asked to what extend they were agree or disagree to three statements: (1) "Social distancing can protect you from COVID-19"; (b) "Social distancing can protect your child or children from COVID-19 (if any)"; and (3) "Social distancing can protect your parents from COVID-19". The possible responses for each statement were in 5-Likert scale from "Strongly agree", "Agree", "Neither agree nor disagree", "Disagree" and "Strongly disagree". For statistical purpose, the responses of the individual were classified as "Disagree" (those who answered disagree and strongly disagree), "Neutral" (neither agree nor disagree) and "Agree" (those who responded agree or strongly agree).

\section{Statistical analysis}

For each type of hypothetical COVID-19 vaccine, a two-step logistic regression was used to determine the associated explanatory variables. During the first step, univariate analysis, the odds ratios (ORs) and the 95\% confidence interval (95\%CI) were calculated separately (i.e., known as crude OR). In the next step, all explanatory variables with a p-value $<0.05$ during the univariate were included and ORs (adjusted ORs and 95\%CIs) were calculated together. For each explanatory variable, a reference group was pre-determined. All analyses were conducted using SPSS software (SPSS Inc., Chicago, IL, USA).

\section{Results}

\section{Demographic characteristics}

A total of 1,337 respondents were included in the final analysis of which the highest percentage originated from India (21.1\%), followed by Pakistan (12.9\%) and Sudan (11.2\%). The characteristics of the respondents are presented in (Table 1). Briefly, more than half of the respondents belonged to 21-30 age group and less than $5 \%$ of them aged older than 51 years old. There was a small gender disproportional; $43.3 \%$ and $56.7 \%$ for male and female, respectively. Most of the respondents (82.9\%) were living in the urban areas and more than half of the respondents (52.2\%) were Muslims, followed by Hindu (15.9\%). Based on self-reported information on chronic comorbidities, there were 5.2\%, 2.9\%, 2.3\% and 5.5\% of the respondents who reported having hypertension, diabetes mellitus, heart disease and pulmonary disease, respectively.

\section{COVID-19 vaccine acceptance rates}

The COVID-19 vaccine acceptance rates were observed to be influenced by the vaccine based on the efficacy and safety profiles. At the worst scenario provided (50\% efficacy and $5 \%$ risk of side effects), the acceptance rate was $58.3 \%(784 / 1337)$ (Table 1). The acceptance rate increased to $80.1 \%(1,078 / 1337)$ for the scenario where the vaccine has a $75 \%$ efficacy and $5 \%$ side effects profile. In the scenario where vaccine efficacy was $75 \%$, the acceptance rate was only $55.6 \%$ when the safety profile was lower (the chance for having the side effects was 20\%). When the vaccine efficacy increased to $95 \%$ with $20 \%$ risk of side effects, the acceptance rate was $74.0 \%$. Across the four vaccine profiles, Brazil and Chile had the highest acceptance rate compared to all other countries (Figure 1). 
A $95 \%$ efficacy; $20 \%$ side effects $\overrightarrow{0 \quad 100}$
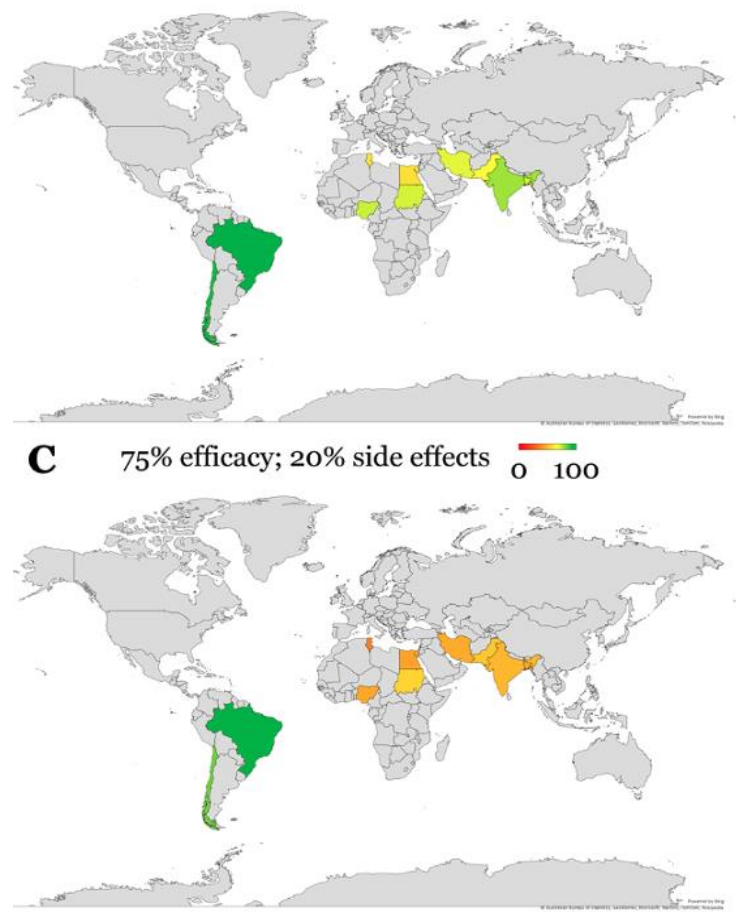

B $75 \%$ efficacy; $5 \%$ side effects $\overrightarrow{0} 100$
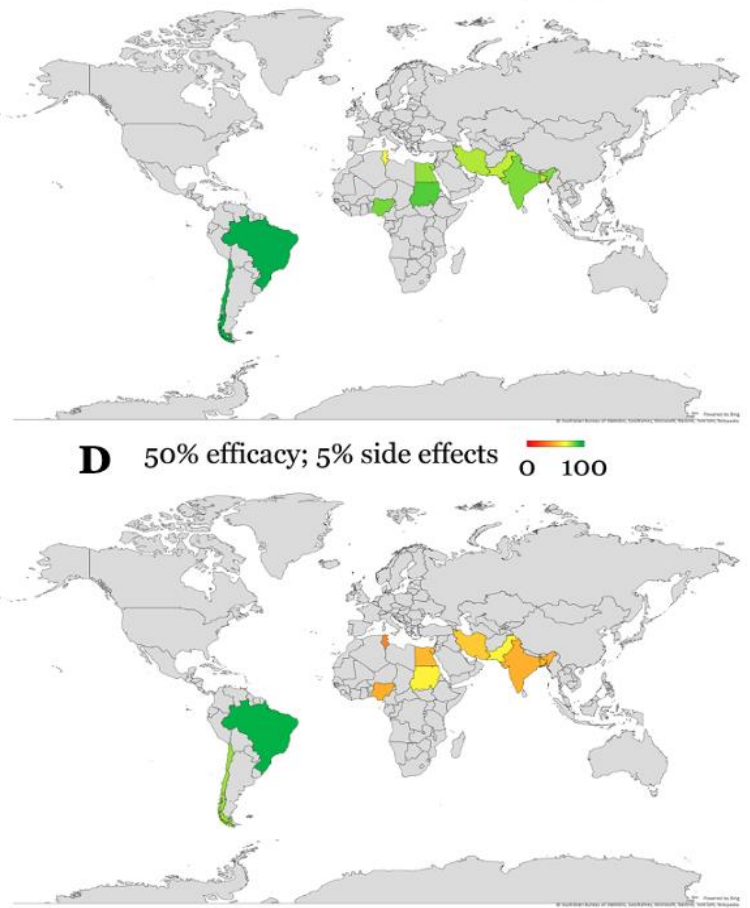

Figure 1. COVID-19 vaccine acceptance rates in the ten countries involved in the study using the four scenarios of varying vaccine efficacy and safety. Color represents the acceptance rate for a COVID-19 vaccine, from o to $100 \%$.

\section{Vaccine acceptance and its explanatory variables}

Data suggested the country, age group, monthly household income, religion, having comorbidities such as hypertension, heart disease or pulmonary disease, changes in salary during the COVID-19 pandemic, the participants' view on vaccination benefits and having flu vaccination in the past 12 months were associated with vaccine acceptance in varying degrees (Table 2). Among of them, some explanatory variables were associated with all four vaccine profiles. The respondents who agreed that vaccines are important for their health had higher chance to accept COVID-19 vaccine with OR: 7.36; 95\%CI: 2.82, 19.22 and OR: 5.32; 95\%CI: $1.74,16.25$ for the best (Vaccine B, 75\% efficacy and $5 \%$ side effects) and the worst hypothetical COVID-19 vaccine (Vaccine D, 50\% efficacy and 20\% side effects), respectively. Compared to those who did not agree that all recommended vaccines are beneficial for their health, those who acknowledged the benefit of the vaccine for their health had higher odds of accepting all vaccine profiles; OR: 2.05 and OR: 2.40 for the best (Vaccine B) and worst vaccine (Vaccine D) scenarios, respectively. Compared to those who believe that new vaccines carry more risks than older vaccines, those who did not hold such a belief had approximately two times higher odds of accepting the COVID-19 vaccine with OR ranging between 1.99 and 2.11 for all four vaccine profiles.

In addition, those who believed that the information provided by the government about vaccines are reliable and trustworthy also had higher odds of accepting the vaccine compared to those who believed that the information is not reliable and trustworthy with OR ranging between 1.48 and 1.70 for different vaccine profiles. Having flu vaccination in the past 12 months was correlated with an increased chance for accepting the COVID-19 vaccine approximately 2 times; the ORs varied among the vaccine profiles ranging between 1.71 and 2.30 (Table 2). 
Table 1. COVID-19 vaccine acceptance rates ( $n=1337)$

\begin{tabular}{|c|c|c|c|c|c|}
\hline \multirow[t]{2}{*}{ Variable } & \multirow[t]{2}{*}{$n(\%)$} & \multicolumn{4}{|c|}{ Vaccine acceptance rate } \\
\hline & & $\begin{array}{l}95 \% \text { vaccine efficacy } \\
20 \% \text { side effect (A) }\end{array}$ & $\begin{array}{l}75 \% \text { vaccine efficacy } \\
5 \% \text { side effect (B) }\end{array}$ & $\begin{array}{l}75 \% \text { vaccine efficacy } \\
20 \% \text { side effect (C) }\end{array}$ & $\begin{array}{l}50 \% \text { vaccine efficacy } \\
5 \% \text { side effect (D) }\end{array}$ \\
\hline Acceptance & $1337(100.0)$ & $990(74.0)$ & $1,078(80.1)$ & $744(55.6)$ & $784(58.3)$ \\
\hline \multicolumn{6}{|l|}{ Country } \\
\hline Pakistan & $173(12.9)$ & $116(67.1)$ & $127(73.4)$ & $94(54.3)$ & $112(64.7)$ \\
\hline Brazil & $106(7.9)$ & $102(96.2)$ & $104(98.1)$ & $101(95 \cdot 3)$ & $101(95 \cdot 3)$ \\
\hline Chile & $103(7.7)$ & $94(91.3)$ & $98(95.1)$ & $85(82.5)$ & $80(77.7)$ \\
\hline Egypt & $83(6.2)$ & $50(60.2)$ & $65(78.3)$ & $38(45.8)$ & $42(50.6)$ \\
\hline India & $282(21.1)$ & $217(77.0)$ & $227(80.5)$ & $142(50.4)$ & $138(48.9)$ \\
\hline Iran & $109(8.2)$ & $76(69.7)$ & $82(75.2)$ & $52(47.7)$ & $60(55.0)$ \\
\hline Nigeria & $124(9.3)$ & $90(72.6)$ & $101(81.5)$ & $58(46.8)$ & $60(48.4)$ \\
\hline Bangladesh & $106(7.9)$ & $76(71.7)$ & $78(73.6)$ & $51(48.1)$ & $56(52.8)$ \\
\hline Sudan & $150(11.2)$ & $107(71.3)$ & $128(85.3)$ & $86(57.3)$ & $96(64.0)$ \\
\hline \multirow{2}{*}{\multicolumn{6}{|c|}{ Age group (year) }} \\
\hline & & & & & \\
\hline$<20$ & $207(15.5)$ & $157(75.8)$ & $168(81.2)$ & $126(60.9)$ & $133(64.3)$ \\
\hline $21-30$ & $780(58.3)$ & $572(73.3)$ & $638(81.8)$ & $424(54.4)$ & $451(57.8)$ \\
\hline $31-40$ & $210(15.7)$ & $149(71.0)$ & $159(75.7)$ & $112(53.3)$ & $115(54.8)$ \\
\hline $41-50$ & $93(7.0)$ & $76(81.7)$ & $75(80.6)$ & $49(52.7)$ & $49(52.7)$ \\
\hline$>51$ & $47(3.5)$ & $36(76.6)$ & $38(80.9)$ & $33(70.2)$ & $36(76.6)$ \\
\hline \multicolumn{6}{|l|}{ Gender } \\
\hline Male & $579(43.3)$ & $430(74.3)$ & $461(79.6)$ & $325(56.1)$ & $351(60.6)$ \\
\hline Female & $758(56.7)$ & $560(73.9)$ & $617(81.4)$ & $419(55 \cdot 3)$ & $433(57.1)$ \\
\hline \multicolumn{6}{|l|}{ Urbanicity } \\
\hline Rural & $228(17.1)$ & $164(71.9)$ & $169(74.1)$ & $118(51.8)$ & $122(53.5)$ \\
\hline Urban & $1109(82.9)$ & $826(74.5)$ & $909(82.0)$ & $626(56.4)$ & $662(59.7)$ \\
\hline \multicolumn{6}{|l|}{ Monthly household income (USD) } \\
\hline$<500$ & $488(36.5)$ & $338(69.3)$ & $375(76.8)$ & $243(49.8)$ & $274(56.1)$ \\
\hline $500-999$ & $267(20.0)$ & $198(74.2)$ & $216(80.9)$ & $147(55.1)$ & $151(56.6)$ \\
\hline $1,000-1,999$ & $175(13.1)$ & $132(75.4)$ & $147(84.0)$ & $103(58.9)$ & $105(60.0)$ \\
\hline $2,000-2,999$ & $128(9.6)$ & $94(73.4)$ & $103(80.5)$ & $70(54.7)$ & $69(53.9)$ \\
\hline $3,000-4,999$ & $104(7.8)$ & $81(77.9)$ & $97(93.3)$ & $73(70.2)$ & $78(75.0)$ \\
\hline $5,000-7,999$ & $85(6.4)$ & $68(80.0)$ & $69(81.2)$ & $47(55.3)$ & $51(60.0)$ \\
\hline$\geq 8,000$ & $90(6.7)$ & $79(87.8)$ & $71(78.9)$ & $61(67.8)$ & $56(62.2)$ \\
\hline \multicolumn{6}{|l|}{ Religion } \\
\hline Islam & $698(52.2)$ & $473(67.8)$ & $534(76.5)$ & $344(49.3)$ & $385(55.2)$ \\
\hline Christian/Protestant/Methodist/Lutheran/Baptist & $140(10.5)$ & $108(77.1)$ & $115(82.1)$ & $77(55.0)$ & $79(56.4)$ \\
\hline Catholic & $118(8.8)$ & $98(83.1)$ & $104(88.1)$ & $83(70.3)$ & $82(69.5)$ \\
\hline Hindu & $212(15.9)$ & $169(79.7)$ & $178(84.0)$ & $112(52.8)$ & $113(53.3)$ \\
\hline Atheist or agnostic & $127(9.5)$ & $106(83.5)$ & $113(89.0)$ & $98(77.2)$ & $95(74.8)$ \\
\hline
\end{tabular}




\begin{tabular}{|c|c|c|c|c|c|}
\hline \multirow[t]{2}{*}{ Variable } & \multirow[t]{2}{*}{$n(\%)$} & \multicolumn{4}{|c|}{ Vaccine acceptance rate } \\
\hline & & $\begin{array}{l}95 \% \text { vaccine efficacy } \\
20 \% \text { side effect }(\mathrm{A})\end{array}$ & $\begin{array}{l}75 \% \text { vaccine efficacy } \\
5 \% \text { side effect }(\mathrm{B})\end{array}$ & $\begin{array}{l}75 \% \text { vaccine efficacy } \\
20 \% \text { side effect }(C)\end{array}$ & $\begin{array}{l}50 \% \text { vaccine efficacy } \\
5 \% \text { side effect (D) }\end{array}$ \\
\hline Others & $42(3.1)$ & $36(85.7)$ & $34(81.0)$ & $30(71.4)$ & $30(71.4)$ \\
\hline \multicolumn{6}{|l|}{ Healthcare-related job } \\
\hline No & $706(52.8)$ & $513(72.7)$ & $575(81.4)$ & $391(55.4)$ & $422(59.8)$ \\
\hline Yes & $631(47.2)$ & $477(75.6)$ & $503(79.7)$ & $353(55.9)$ & $362(57.4)$ \\
\hline \multicolumn{6}{|l|}{ Have hypertension } \\
\hline $\mathrm{No}^{\mathrm{a}}$ & $917(68.6)$ & $693(75.6)$ & $733(79.9)$ & $526(57.4)$ & $539(58.8)$ \\
\hline Yes ${ }^{b}$ & $70(5.2)$ & $57(81.4)$ & $58(82.9)$ & $45(64 \cdot 3)$ & $47(67.1)$ \\
\hline Do not know & $350(26.2)$ & $240(68.6)$ & $287(82.0)$ & $173(49.4)$ & $198(56.6)$ \\
\hline \multicolumn{6}{|l|}{ Have diabetes } \\
\hline $\mathrm{No}^{\mathrm{a}}$ & $991(74.1)$ & $742(74.9)$ & $801(80.8)$ & $560(56.5)$ & $584(58.9)$ \\
\hline Yes $^{b}$ & $39(2.9)$ & $30(76.9)$ & $31(79.5)$ & $25(64.1)$ & $28(71.8)$ \\
\hline Do not know & $307(23.0)$ & $218(71.0)$ & $246(80.1)$ & $159(51.8)$ & $172(56.0)$ \\
\hline \multicolumn{6}{|l|}{ Have heart disease } \\
\hline $\mathrm{No}^{\mathrm{a}}$ & $909(68.0)$ & $692(76.1)$ & $734(80.7)$ & $524(57.6)$ & $539(59.3)$ \\
\hline Yes $^{b}$ & $31(2.3)$ & $23(74.2)$ & $23(74.2)$ & $19(61.3)$ & $21(67.7)$ \\
\hline Do not know & $397(29.7)$ & $275(69.3)$ & $321(80.9)$ & $201(50.6)$ & $224(56.4)$ \\
\hline \multicolumn{6}{|l|}{ Have pulmonary disease } \\
\hline $\mathrm{No}^{\text {a }}$ & $865(64.7)$ & $653(75.5)$ & $700(80.9)$ & $502(58.0)$ & $521(60.2)$ \\
\hline Yes $^{b}$ & $73(5.5)$ & $55(75 \cdot 3)$ & $58(79.5)$ & $41(56.2)$ & $48(65.8)$ \\
\hline Do not know & $399(29.8)$ & $282(70.7)$ & $320(80.2)$ & $201(50.4)$ & $215(53.9)$ \\
\hline \multicolumn{6}{|l|}{ Occupation } \\
\hline Self-employed & $125(9.3)$ & $94(75.2)$ & $102(81.6)$ & $73(58.4)$ & $76(60.8)$ \\
\hline Employed for wages & $342(25.6)$ & $262(76.6)$ & $268(78.4)$ & $185(54.1)$ & $187(54.7)$ \\
\hline Out of work & $57(4.3)$ & $47(82.5)$ & $50(87.7)$ & $34(59.6)$ & $35(61.4)$ \\
\hline Homemaker & $23(1.7)$ & $18(78.3)$ & $20(87.0)$ & $16(69.6)$ & $14(60.9)$ \\
\hline Student & $773(57.8)$ & $558(72.2)$ & $626(81.0)$ & $427(55.2)$ & $459(59.4)$ \\
\hline Retired or unable to work & $17(1.3)$ & $11(64.7)$ & $12(70.6)$ & $9(52.9)$ & $13(76.5)$ \\
\hline \multicolumn{6}{|c|}{$\begin{array}{l}\text { Has how much your work changed as a result of the COVID- } \\
19 \text { pandemic? }\end{array}$} \\
\hline I work fewer hours & $232(17.4)$ & $167(72.0)$ & $179(77.2)$ & $117(50.4)$ & $140(60.3)$ \\
\hline No change and not applicable (not working) & $816(61.0)$ & $601(73.7)$ & $665(81.5)$ & $453(55 \cdot 5)$ & $471(57.7)$ \\
\hline I work more hours & $253(18.9)$ & $196(77.5)$ & $202(79.8)$ & $155(61.3)$ & $152(60.1)$ \\
\hline I was let go from my job & $36(2.7)$ & $26(72.2)$ & $32(88.9)$ & $19(52.8)$ & $21(58.3)$ \\
\hline \multirow{2}{*}{\multicolumn{6}{|c|}{$\begin{array}{l}\text { Has how much your salary changed as a result of the } \\
\text { COVID-19 pandemic? }\end{array}$}} \\
\hline & & & & & \\
\hline I am getting paid less & $384(28.7)$ & $264(68.8)$ & $308(80.2)$ & $194(50.5)$ & $216(56.3)$ \\
\hline I am getting paid more & $91(6.8)$ & $74(81.3)$ & $76(83.5)$ & $61(67.0)$ & $61(67.0)$ \\
\hline No changes & $862(64.5)$ & $652(75.6)$ & $694(80.5)$ & $489(56.7)$ & $507(58.8)$ \\
\hline \multicolumn{6}{|l|}{ Vaccines are important for my health } \\
\hline Disagree or strongly disagree & $18(1.3)$ & $9(50.0)$ & $7(38.9)$ & $6(33.3)$ & $4(22.2)$ \\
\hline Neither agree nor disagree & $125(9.3)$ & $79(63.2)$ & $87(69.6)$ & $52(41.6)$ & $60(48.0)$ \\
\hline Agree or strongly agree & $1194(89.3)$ & $902(75.5)$ & $984(82.4)$ & $686(57.5)$ & $720(60.3)$ \\
\hline
\end{tabular}




\begin{tabular}{|c|c|c|c|c|c|}
\hline \multirow[t]{2}{*}{ Variable } & \multirow[t]{2}{*}{$n(\%)$} & \multicolumn{4}{|c|}{ Vaccine acceptance rate } \\
\hline & & $\begin{array}{l}95 \% \text { vaccine efficacy } \\
20 \% \text { side effect }(\mathrm{A})\end{array}$ & $\begin{array}{l}75 \% \text { vaccine efficacy } \\
5 \% \text { side effect }(\mathrm{B})\end{array}$ & $\begin{array}{l}75 \% \text { vaccine efficacy } \\
20 \% \text { side effect }(C)\end{array}$ & $\begin{array}{l}50 \% \text { vaccine efficacy } \\
5 \% \text { side effect }(\mathrm{D})\end{array}$ \\
\hline \multicolumn{6}{|c|}{ All routine vaccines recommended by the healthcare } \\
\hline Disagree or strongly disagree & $43(3.2)$ & $25(58.1)$ & $30(69.8)$ & $16(37.2)$ & $17(39.5)$ \\
\hline Neither agree nor disagree & $187(14.0)$ & $122(65.2)$ & $134(71.7)$ & $78(41.7)$ & $91(48.7)$ \\
\hline Agree or strongly agree & $1107(82.8)$ & $843(76.2)$ & $914(82.6)$ & $650(58.7)$ & $676(61.1)$ \\
\hline \multicolumn{6}{|c|}{ New vaccines carry more risks than older vaccines } \\
\hline Agree or strongly agree & $477(35.7)$ & $329(69.0)$ & $365(76.5)$ & $236(49.5)$ & $256(53.7)$ \\
\hline Neither agree nor disagree & $529(39.6)$ & $389(73.5)$ & $425(80.3)$ & $285(53.9)$ & $297(56.1)$ \\
\hline Disagree or strongly disagree & $331(24.8)$ & $272(82.2)$ & $288(87.0)$ & $223(67.4)$ & $231(69.8)$ \\
\hline \multicolumn{6}{|c|}{$\begin{array}{l}\text { The information I receive about vaccines from the } \\
\text { government is reliable and trustworthy }\end{array}$} \\
\hline Disagree or strongly disagree & $222(16.6)$ & $153(68.9)$ & $169(76.1)$ & $115(51.8)$ & $117(52.7)$ \\
\hline Neither agree nor disagree & $422(31.6)$ & $289(68.5)$ & $337(79.9)$ & $201(47.6)$ & $230(54.5)$ \\
\hline Agree or strongly agree & $693(51.8)$ & $548(79.1)$ & $572(82.5)$ & $428(61.8)$ & $437(63.1)$ \\
\hline \multicolumn{6}{|c|}{ Getting vaccines is a good way to protect me from disease } \\
\hline Disagree or strongly disagree & $27(2.0)$ & $15(55.6)$ & $15(55.6)$ & $9(33.3)$ & $6(22.2)$ \\
\hline Neither agree nor disagree & $125(9.3)$ & $74(59.2)$ & $80(64.0)$ & $53(42.4)$ & $62(49.6)$ \\
\hline Agree or strongly agree & $1185(88.6)$ & $901(76.0)$ & $983(83.0)$ & $682(57.6)$ & $716(60.4)$ \\
\hline \multicolumn{6}{|c|}{ Social distancing can protect yourself from COVID-19 } \\
\hline Disagree or strongly disagree & $18(1.3)$ & $11(61.1)$ & $12(66.7)$ & $4(22.2)$ & $11(61.1)$ \\
\hline Neither agree nor disagree & $52(3.9)$ & $34(65.4)$ & $36(69.2)$ & $23(44.2)$ & $22(42.3)$ \\
\hline Agree or strongly agree & $1267(94.8)$ & $945(74.6)$ & $1030(81.3)$ & $717(56.6)$ & $751(59.3)$ \\
\hline \multicolumn{6}{|c|}{ Social distancing can protect your child or children from } \\
\hline Disagree or strongly disagree & $28(2.1)$ & $15(53.6)$ & $20(71.4)$ & $11(39.3)$ & $13(46.4)$ \\
\hline Neither agree nor disagree & $58(4.3)$ & $42(72.4)$ & $41(70.7)$ & $29(50.0)$ & $33(56.9)$ \\
\hline Agree or strongly agree & $1251(93.6)$ & $933(74.6)$ & $1017(81.3)$ & $704(56.3)$ & $738(59.0)$ \\
\hline \multicolumn{6}{|c|}{ Social distancing can protect your parents from COVID-19 } \\
\hline Disagree or strongly disagree & $21(1.6)$ & $13(61.9)$ & $14(66.7)$ & $6(28.6)$ & $10(47.6)$ \\
\hline Neither agree nor disagree & $45(3.4)$ & $28(62.2)$ & $31(68.9)$ & $18(40.0)$ & $22(48.9)$ \\
\hline \multirow{2}{*}{\multicolumn{6}{|c|}{ Having flu vaccination during the past 12 months }} \\
\hline & & & & & \\
\hline No & $1054(78.8)$ & $756(71.7)$ & $833(79.0)$ & $543(51.5)$ & $582(55.2)$ \\
\hline Yes & $283(21.2)$ & $234(82.7)$ & 245 (86.6) & 201 (71.0) & $202(71.4)$ \\
\hline
\end{tabular}


Table 2. Determinants associated with COVID-19 vaccine acceptance $(n=1337)$

\begin{tabular}{|c|c|c|c|c|c|c|c|c|}
\hline \multirow[t]{2}{*}{ Variable } & \multicolumn{2}{|l|}{$\begin{array}{l}95 \% \text { vaccine efficacy } \\
20 \% \text { side effect }(A)\end{array}$} & \multicolumn{2}{|l|}{$\begin{array}{l}75 \% \text { vaccine efficacy } \\
5 \% \text { side effect (B) }\end{array}$} & \multicolumn{2}{|l|}{$\begin{array}{l}75 \% \text { vaccine efficacy } \\
20 \% \text { side effect }(\mathrm{C})\end{array}$} & \multicolumn{2}{|l|}{$\begin{array}{l}50 \% \text { vaccine efficacy } \\
5 \% \text { side effect (D) }\end{array}$} \\
\hline & OR (95\% CI) & $p$ & OR (95\% CI) & $p$ & OR (95\% CI) & $p$ & OR (95\% CI) & $p$ \\
\hline \multicolumn{9}{|l|}{ Country } \\
\hline Pakistan (Reference, $R$ ) & 1 & & 1 & & 1 & & 1 & \\
\hline Brazil & $12.53(4.39,35.74)$ & $<0.001$ & $18.84(4.47,79.44)$ & $<0.001$ & $16.98(6.59,43.74)$ & $<0.001$ & $11.00(4.25,28.46)$ & $<0.001$ \\
\hline Chile & $5.13(2.42,10.91)$ & $<0.001$ & $7.10(2.72,18.54)$ & $<0.001$ & $3.97(2.20,7.16)$ & $<0.001$ & $1.89(1.08,3.31)$ & 0.025 \\
\hline Egypt & $0.75(0.43,1.28)$ & 0.286 & $1.31(0.70,2.44)$ & 0.397 & $0.71(0.42,1.20)$ & 0.201 & $0.56(0.33,0.95)$ & 0.031 \\
\hline India & $1.64(1.08,2.50)$ & 0.021 & $1.50(0.96,2.34)$ & 0.078 & $0.85(0.58,1.25)$ & 0.410 & $0.52(0.35,0.77)$ & 0.001 \\
\hline Iran & $1.13(0.68,1.90)$ & 0.639 & $1.10(0.63,1.91)$ & 0.734 & $0.77(0.47,1.24)$ & 0.278 & $0.67(0.41,1.09)$ & 0.105 \\
\hline Nigeria & $1.30(0.78,2.16)$ & 0.309 & $1.59(0.90,2.80)$ & 0.107 & $0.74(0.47,1.17)$ & 0.199 & $0.51(0.32,0.82)$ & 0.005 \\
\hline Bangladesh & $1.25(0.73,2.11)$ & 0.417 & $1.01(0.58,1.75)$ & 0.974 & $0.78(0.48,1.27)$ & 0.313 & $0.61(0.37,1.00)$ & 0.049 \\
\hline Sudan & $1.22(0.76,1.97)$ & 0.407 & $2.11(1.20,3.71)$ & 0.010 & $1.13(0.73,1.76)$ & 0.589 & $0.97(0.61,1.53)$ & 0.890 \\
\hline Tunisia & $0.78(0.47,1.30)$ & 0.343 & $0.75(0.44,1.28)$ & 0.284 & $0.49(0.29,0.80)$ & 0.005 & $0.34(0.21,0.57)$ & $<0.001$ \\
\hline \multicolumn{9}{|l|}{ Age group (year) } \\
\hline$<20(R)$ & 1 & & 1 & & 1 & & 1 & \\
\hline $21-30$ & $0.88(0.61,1.25)$ & 0.465 & $1.04(0.70,1.55)$ & 0.834 & $0.77(0.56,1.05)$ & 0.094 & $0.76(0.56,1.05)$ & 0.095 \\
\hline $31-40$ & $0.78(0.50,1.20)$ & 0.259 & $0.72(0.45,1.16)$ & 0.178 & $0.74(0.50,1.08)$ & 0.120 & $0.67(0.46,1.00)$ & 0.049 \\
\hline $41-50$ & $1.42(0.77,2.63)$ & 0.260 & $0.97(0.52,1.80)$ & 0.916 & $0.72(0.44,1.17)$ & 0.184 & $0.62(0.38,1.02)$ & 0.059 \\
\hline$>51$ & $1.04(0.49,2.20)$ & 0.913 & $0.98(0.44,2.19)$ & 0.961 & $1.52(0.76,3.01)$ & 0.234 & $1.82(0.88,3.79)$ & 0.109 \\
\hline \multicolumn{9}{|l|}{ Gender } \\
\hline Male $(R)$ & 1 & & 1 & & 1 & & 1 & \\
\hline Female & $0.98(0.77,1.26)$ & 0.873 & $1.12(0.85,1.47)$ & 0.415 & $0.97(0.78,1.20)$ & 0.755 & $0.87(0.69,1.08)$ & 0.198 \\
\hline \multicolumn{9}{|l|}{ Urbanicity } \\
\hline Rural $(R)$ & 1 & & 1 & & 1 & & 1 & \\
\hline Urban & $1.14(0.83,1.57)$ & 0.424 & $1.59(1.14,2.22)$ & 0.007 & $1.21(0.91,1.61)$ & 0.194 & $1.29(0.97,1.71)$ & 0.085 \\
\hline \multicolumn{9}{|l|}{ Monthly household income (USD) } \\
\hline$<500(R)$ & 1 & & 1 & & 1 & & 1 & \\
\hline $500-999$ & $1.27(0.91,1.78)$ & 0.157 & $1.28(0.88,1.85)$ & 0.197 & $1.24(0.92,1.67)$ & 0.167 & $1.02(0.75,1.37)$ & 0.914 \\
\hline $1,000-1,999$ & $1.36(0.92,2.02)$ & 0.124 & $1.58(1.00,2.50)$ & 0.048 & $1.44(1.02,2.05)$ & 0.040 & $1.17(0.82,1.67)$ & 0.377 \\
\hline 2,000-2,999 & $1.23(0.79,1.90)$ & 0.359 & $1.24(0.76,2.02)$ & 0.382 & $1.22(0.82,1.80)$ & 0.325 & $0.91(0.62,1.35)$ & 0.650 \\
\hline $3,000-4,999$ & $1.56(0.95,2.58)$ & 0.081 & $4.18(1.89,9.25)$ & $<0.001$ & $2.37(1.51,3.75)$ & $<0.001$ & $2.34(1.45,3.78)$ & $<0.001$ \\
\hline $5,000-7,999$ & $1.78(1.01,3.14)$ & 0.047 & $1.30(0.73,2.33)$ & 0.379 & $1.25(0.79,1.98)$ & 0.350 & $1.17(0.73,1.87)$ & 0.509 \\
\hline$\geq 8,000$ & $3.19(1.65,6.16)$ & 0.001 & $1.13(0.65,1.95)$ & 0.671 & $2.12(1.32,3.42)$ & 0.002 & $1.29(0.81,2.04)$ & 0.285 \\
\hline \multicolumn{9}{|l|}{ Religion } \\
\hline $\operatorname{Islam}(R)$ & 1 & & 1 & & 1 & & 1 & \\
\hline $\begin{array}{l}\text { Christian/Protestant/Methodist/Lutheran } \\
\text { /Baptist }\end{array}$ & $1.61(1.05,2.46)$ & 0.029 & $1.41(0.89,2.25)$ & 0.147 & $1.26(0.87,1.81)$ & 0.218 & $1.05(0.73,1.52)$ & 0.782 \\
\hline Catholic & $2.33(1.41,3.87)$ & 0.001 & $2.28(1.27,4.09)$ & 0.006 & $2.44(1.60,3.72)$ & $<0.001$ & $1.85(1.22,2.82)$ & 0.004 \\
\hline Hindu & $1.87(1.29,2.71)$ & 0.001 & $1.61(1.07,2.41)$ & 0.022 & $1.15(0.85,1.57)$ & 0.366 & $0.93(0.68,1.26)$ & 0.635 \\
\hline Atheist or agnostic & $2.40(1.47,3.94)$ & 0.001 & $2.48(1.39,4.44)$ & 0.002 & $3.48(2.24,5.40)$ & $<0.001$ & $2.41(1.57,3.70)$ & $<0.001$ \\
\hline Others & $2.85(1.19,6.87)$ & 0.019 & $1.31(0.59,2.88)$ & 0.509 & $2.57(1.30,5.11)$ & 0.007 & $2.03(1.02,4.04)$ & 0.043 \\
\hline
\end{tabular}




\begin{tabular}{|c|c|c|c|c|c|c|c|c|}
\hline \multirow[t]{2}{*}{ Variable } & \multicolumn{2}{|c|}{$\begin{array}{l}95 \% \text { vaccine efficacy } \\
20 \% \text { side effect }(\mathrm{A})\end{array}$} & \multicolumn{2}{|c|}{$\begin{array}{l}75 \% \text { vaccine efficacy } \\
5 \% \text { side effect }(\mathrm{B})\end{array}$} & \multicolumn{2}{|c|}{$\begin{array}{l}75 \% \text { vaccine efficacy } \\
20 \% \text { side effect }(\mathrm{C})\end{array}$} & \multicolumn{2}{|c|}{$\begin{array}{l}50 \% \text { vaccine efficacy } \\
5 \% \text { side effect }(D)\end{array}$} \\
\hline & OR (95\% CI) & $p$ & OR (95\% CI) & $p$ & OR (95\% CI) & $p$ & OR (95\% CI) & $p$ \\
\hline \multicolumn{9}{|l|}{ Healthcare-related job } \\
\hline No $(R)$ & 1 & & 1 & & 1 & & 1 & \\
\hline Yes & $1.17(0.91,1.49)$ & 0.222 & $0.90(0.68,1.17)$ & 0.424 & $1.02(0.82,1.27)$ & 0.837 & $0.91(0.73,1.13)$ & 0.373 \\
\hline \multicolumn{9}{|l|}{ Have hypertension } \\
\hline $\mathrm{No}^{\mathrm{a}}(R)$ & 1 & & 1 & & 1 & & 1 & \\
\hline Yes ${ }^{b}$ & $1.42(0.76,2.64)$ & 0.271 & $1.21(0.64,2.31)$ & 0.555 & $1.34(0.81,2.20)$ & 0.259 & $1.43(0.86,2.40)$ & 0.172 \\
\hline Do not know & $0.71(0.54,0.93)$ & 0.012 & $1.14(0.83,1.57)$ & 0.407 & $0.73(0.57,0.93)$ & 0.011 & $0.91(0.71,1.17)$ & 0.476 \\
\hline \multicolumn{9}{|l|}{ Have diabetes } \\
\hline $\mathrm{No}^{\mathrm{a}}(R)$ & 1 & & 1 & & 1 & & 1 & \\
\hline Yes b & $1.12(0.52,2.39)$ & 0.772 & $0.92(0.42,2.03)$ & 0.835 & $1.37(0.71,2.68)$ & 0.350 & $1.77(0.87,3.61)$ & 0.113 \\
\hline Do not know & $0.82(0.62,1.09)$ & 0.178 & $0.96(0.69,1.32)$ & 0.787 & $0.83(0.64,1.07)$ & 0.147 & $0.89(0.69,1.15)$ & 0.367 \\
\hline \multicolumn{9}{|l|}{ Have heart disease } \\
\hline $\mathrm{No}^{\mathrm{a}}(R)$ & 1 & & 1 & & 1 & & 1 & \\
\hline Yes ${ }^{b}$ & $0.90(0.40,2.05)$ & 0.804 & $0.69(0.30,1.56)$ & 0.367 & $1.16(0.56,2.43)$ & 0.686 & $1.44(0.67,3.10)$ & 0.348 \\
\hline Do not know & $0.71(0.54,0.92)$ & 0.009 & $1.01(0.75,1.34)$ & 0.964 & $0.75(0.60,0.96)$ & 0.019 & $0.89(0.70,1.13)$ & 0.333 \\
\hline \multicolumn{9}{|l|}{ Have pulmonary disease } \\
\hline $\mathrm{No}^{\mathrm{a}}(R)$ & 1 & & 1 & & 1 & & 1 & \\
\hline Yes b & $0.99(0.57,1.73)$ & 0.977 & $0.91(0.50,1.65)$ & 0.759 & $0.93(0.57,1.50)$ & 0.756 & $1.27(0.77,2.10)$ & 0.355 \\
\hline Do not know & $0.78(0.60,1.02)$ & 0.070 & $0.96(0.71,1.29)$ & 0.762 & $0.73(0.58,0.93)$ & 0.011 & $0.77(0.61,0.98)$ & 0.034 \\
\hline \multicolumn{9}{|l|}{ Occupation } \\
\hline Self-employed $(R)$ & 1 & & 1 & & 1 & & 1 & \\
\hline Employed for wages & $1.08(0.67,1.74)$ & 0.752 & $0.82(0.49,1.37)$ & 0.446 & $0.84(0.56,1.27)$ & 0.408 & $0.78(0.51,1.18)$ & 0.238 \\
\hline $\begin{array}{l}\text { Out of work for less } 1 \text { year AND more than } \\
1 \text { year }\end{array}$ & $1.55(0.70,3.43)$ & 0.279 & $1.61(0.65,4.01)$ & 0.305 & $1.05(0.56,1.99)$ & 0.874 & $1.03(0.54,1.95)$ & 0.938 \\
\hline Homemaker & $1.19(0.41,3.46)$ & 0.753 & $1.50(0.41,5.49)$ & 0.537 & $1.63(0.63,4.24)$ & 0.318 & $1.00(0.40,2.49)$ & 0.995 \\
\hline Student & $0.86(0.55,1.32)$ & 0.484 & $0.96(0.59,1.56)$ & 0.870 & $0.88(0.60,1.29)$ & 0.509 & $0.94(0.64,1.39)$ & 0.764 \\
\hline Retired or unable to work & $0.61(0.21,1.77)$ & 0.359 & $0.54(0.17,1.69)$ & 0.290 & $0.80(0.29,2.22)$ & 0.669 & $2.10(0.65,6.80)$ & 0.218 \\
\hline \multicolumn{9}{|l|}{$\begin{array}{l}\text { Has how much your work changed as a result } \\
\text { of the COVID-19 pandemic? }\end{array}$} \\
\hline I work fewer hours $(R)$ & 1 & & 1 & & 1 & & 1 & \\
\hline No change or not applicable (not working) & $1.09(0.79,1.51)$ & 0.612 & $1.30(0.92,1.86)$ & 0.141 & $1.23(0.92,1.64)$ & 0.170 & $0.90(0.67,1.21)$ & 0.475 \\
\hline I work more hours & $1.34(0.89,2.02)$ & 0.165 & $1.17(0.76,1.81)$ & 0.472 & $1.56(1.08,2.23)$ & 0.017 & $0.99(0.69,1.42)$ & 0.952 \\
\hline I was let go from my job & $1.01(0.46,2.22)$ & 0.979 & $2.37(0.80,7.00)$ & 0.119 & $1.10(0.54,2.22)$ & 0.793 & $0.91(0.45,1.88)$ & 0.819 \\
\hline \multicolumn{9}{|l|}{$\begin{array}{l}\text { Has how much your salary changed as a result } \\
\text { of the COVID-19 pandemic? }\end{array}$} \\
\hline I am getting paid less $(R)$ & 1 & & 1 & & 1 & & 1 & \\
\hline I am getting paid more & $1.98(1.12,3.50)$ & 0.019 & $1.25(0.68,2.30)$ & 0.472 & $1.99(1.23,3.22)$ & 0.005 & $1.58(0.98,2.56)$ & 0.062 \\
\hline No changes & $1.41(1.08,1.84)$ & 0.011 & $1.02(0.75,1.38)$ & 0.901 & $1.28(1.01,1.63)$ & 0.042 & $1.11(0.87,1.42)$ & 0.397 \\
\hline \multicolumn{9}{|l|}{ Vaccines are important for my health } \\
\hline Disagree or strongly disagree $(R)$ & 1 & & 1 & & 1 & & 1 & \\
\hline Neither agree nor disagree & $1.72(0.64,4.64)$ & 0.286 & $3.60(1.30,9.99)$ & 0.014 & $1.43(0.50,4.04)$ & 0.506 & $3.23(1.01,10.36)$ & 0.049 \\
\hline Agree or strongly agree & $3.09(1.22,7.86)$ & 0.018 & $7.36(2.82,19.22)$ & $<0.001$ & $2.70(1.01,7.24)$ & 0.048 & $5.32(1.74,16.25)$ & 0.003 \\
\hline
\end{tabular}




\begin{tabular}{|c|c|c|c|c|c|c|c|c|}
\hline \multirow[t]{2}{*}{ Variable } & \multicolumn{2}{|c|}{$\begin{array}{l}95 \% \text { vaccine efficacy } \\
20 \% \text { side effect }(\mathrm{A})\end{array}$} & \multicolumn{2}{|c|}{$\begin{array}{l}75 \% \text { vaccine efficacy } \\
5 \% \text { side effect }(\mathrm{B})\end{array}$} & \multicolumn{2}{|c|}{$\begin{array}{l}75 \% \text { vaccine efficacy } \\
20 \% \text { side effect }(\mathrm{C})\end{array}$} & \multicolumn{2}{|c|}{$\begin{array}{l}\text { 50\% vaccine efficacy } \\
5 \% \text { side effect (D) }\end{array}$} \\
\hline & OR (95\% CI) & $p$ & OR (95\% CI) & $p$ & OR (95\% CI) & $p$ & OR (95\% CI) & $p$ \\
\hline \multicolumn{9}{|l|}{$\begin{array}{l}\text { All routine vaccines recommended by the } \\
\text { healthcare workers are beneficial }\end{array}$} \\
\hline Disagree or strongly disagree $(R)$ & 1 & & 1 & & 1 & & 1 & \\
\hline Neither agree nor disagree & $1.35(0.69,2.66)$ & 0.383 & $1.10(0.53,2.26)$ & 0.805 & $1.21(0.61,2.39)$ & 0.588 & $1.45(0.74,2.85)$ & 0.281 \\
\hline Agree or strongly agree & $2.30(1.24,4.28)$ & 0.009 & $2.05(1.05,4.01)$ & 0.035 & $2.40(1.28,4.51)$ & 0.006 & $2.40(1.29,4.47)$ & 0.006 \\
\hline \multicolumn{9}{|l|}{$\begin{array}{l}\text { New vaccines carry more risks than older } \\
\text { vaccines }\end{array}$} \\
\hline Agree or strongly agree $(R)$ & 1 & & 1 & & 1 & & 1 & \\
\hline Neither agree nor disagree & $1.25(0.95,1.64)$ & 0.110 & $1.25(0.93,1.70)$ & 0.141 & $1.19(0.93,1.53)$ & 0.163 & $1.11(0.86,1.42)$ & 0.431 \\
\hline Disagree or strongly disagree & $2.07(1.47,2.92)$ & $<0.001$ & $2.06(1.40,3.02)$ & $<0.001$ & $2.11(1.58,2.82)$ & $<0.001$ & $1.99(1.48,2.58)$ & $<0.001$ \\
\hline \multicolumn{9}{|c|}{$\begin{array}{l}\text { The information I receive about vaccines from } \\
\text { the government is reliable and trustworthy }\end{array}$} \\
\hline Disagree or strongly disagree $(R)$ & 1 & & 1 & & 1 & & 1 & \\
\hline Neither agree nor disagree & $0.98(0.69,1.39)$ & 0.910 & $1.24(0.84,1.84)$ & 0.273 & $0.85(0.61,1.17)$ & 0.314 & $1.08(0.78,1.49)$ & 0.663 \\
\hline Agree or strongly agree & $1.70(1.22,2.39)$ & 0.002 & $1.48(1.03,2.14)$ & 0.035 & $1.50(1.11,2.04)$ & 0.009 & $1.53(1.13,2.08)$ & 0.006 \\
\hline \multicolumn{9}{|c|}{$\begin{array}{l}\text { Getting vaccines is a good way to protect me } \\
\text { from disease }\end{array}$} \\
\hline Disagree or strongly disagree $(R)$ & 1 & & 1 & & 1 & & 1 & \\
\hline Neither agree nor disagree & $1.16(0.50,2.69)$ & 0.728 & $1.42(0.61,3.30)$ & 0.412 & $1.47(0.61,3.53)$ & 0.386 & $3.44(1.30,9.11)$ & 0.013 \\
\hline Agree or strongly agree & $2.54(1.17,5.49)$ & 0.018 & $3.89(1.80,8.44)$ & 0.001 & $2.71(1.21,6.09)$ & 0.016 & $5.34(2.14,13.34)$ & $<0.001$ \\
\hline \multicolumn{9}{|c|}{$\begin{array}{l}\text { Social distancing can protect yourself from } \\
\text { COVID-19 }\end{array}$} \\
\hline Disagree or strongly disagree $(R)$ & 1 & & 1 & & 1 & & 1 & \\
\hline Neither agree nor disagree & $1.20(0.40,3.64)$ & 0.744 & $1.13(0.36,3.53)$ & 0.840 & $2.78(0.80,9.58)$ & 0.106 & $0.47(0.16,1.40)$ & 0.173 \\
\hline Agree or strongly agree & $1.87(0.72,4.86)$ & 0.200 & $2.17(0.81,5.85)$ & 0.124 & $4.56(1.49,13.94)$ & 0.008 & $0.93(0.36,2.41)$ & 0.875 \\
\hline \multicolumn{9}{|c|}{$\begin{array}{l}\text { Social distancing can protect your child or } \\
\text { children from COVID-19 }\end{array}$} \\
\hline Disagree or strongly disagree $(R)$ & 1 & & 1 & & 1 & & 1 & \\
\hline Neither agree nor disagree & $2.28(0.89,5.82)$ & 0.086 & $0.97(0.36,2.61)$ & 0.944 & $1.55(0.62,3.87)$ & 0.352 & $1.52(0.62-3.77)$ & 0.363 \\
\hline Agree or strongly agree & $2.54(1.20,5.40)$ & 0.015 & $1.74(0.76,4.00)$ & 0.193 & $1.99(0.92,4.28)$ & 0.079 & $1.66(0.78-3.52)$ & 0.186 \\
\hline \multicolumn{9}{|c|}{$\begin{array}{l}\text { Social distancing can protect your parents } \\
\text { from COVID-19 }\end{array}$} \\
\hline Disagree or strongly disagree $(R)$ & 1 & & 1 & & 1 & & 1 & \\
\hline Neither agree nor disagree & $1.01(0.35,2.95)$ & 0.980 & $1.11(0.37,3.43)$ & 0.857 & $1.67(0.54,5.10)$ & 0.371 & $1.05(0.37,2.97)$ & 0.923 \\
\hline Agree or strongly agree & $1.81(0.75,4.42)$ & 0.190 & $2.17(0.87,5.44)$ & 0.098 & $3.27(1.26,8.47)$ & 0.015 & $1.59(0.67,3.78)$ & 0.290 \\
\hline \multicolumn{9}{|l|}{$\begin{array}{l}\text { Having flu vaccination during the past } 12 \\
\text { months }\end{array}$} \\
\hline No $(R)$ & 1 & & 1 & & 1 & & 1 & \\
\hline Yes & $1.88(1.35,2.63)$ & $<0.001$ & $1.71(1.18,2.48)$ & 0.005 & $2.31(1.74,3.06)$ & $<0.001$ & $2.02(1.52,2.69)$ & $<0.001$ \\
\hline
\end{tabular}

\section{$\mathrm{R}$ : reference group}




\section{Discussion}

The major result of the current study was the demonstration of how communication about COVID-19 vaccine safety and efficacy can affect its acceptance. The current study collected data from ten various LMICs across three different continents. In the majority of countries, COVID19 vaccine acceptance rates were observed to be higher for the scenarios where vaccine safety and efficacy profiles were superior to other scenarios.

Specifically, at the scenarios of low possibility (5\%) of side effects (e.g., fever or localized pain), with an efficacy of $75 \%$, the vaccine acceptance rates across the ten countries exceeded $67 \%$. Such an acceptance rate is assumed to be close to the lower estimated limit that is necessary to achieve population immunity, with subsequent control of SARS-CoV-2 spread [35, 36]. For the majority of the currently available COVID-19 vaccines, generally high levels of efficacy ( $>80 \%$ ) were reported, and the frequency of side effects was reported at variable levels; however, the majority of which have also reported localized reactions existing for a brief period [37-39]. Therefore, emphasizing that the spread of such messages about the safety of the currently available COVID-19 vaccines can have a positive effect on influencing the public intentions to get vaccinated [40].

In the context of COVID-19 vaccination, the speed with which various vaccine formulas was developed has been linked to high levels of mistrust regarding its safety and subsequent fear of side effects and vaccine hesitancy/rejection $[41,42]$. Thus, the emphasis on the generally high levels of vaccine safety as indicated by the results of clinical trials and the different studies at the general population levels in various settings appears of utmost value to properly address the phenomenon of COVID-19 vaccine hesitancy [43-45].

The current study also showed variable overall levels of COVID-19 vaccine acceptance as follows: the highest acceptance rates were observed in the two South American countries involved in this study (Brazil and Chile), while the lowest levels were observed in Egypt, Tunisia, Iran and Bangladesh. This observation has been previously seen among the Middle East and North Africa countries, where the phenomenon of COVID-19 vaccine hesitancy was more pronounced in various studies [19, 46-48]. Many studies cited concerns about vaccine safety among the study participants as the main reason behind vaccine hesitancy, which adds further support to our findings [46, 49].

For example, in Egypt two previous studies that were conducted in late 2020 and early 2021 among healthcare workers showed high rates of COVID-19 vaccine hesitancy/rejection [50, 51]. The first study found that the absence of enough clinical trials and the fear of side effects of the vaccine (both prevalent among more than $90 \%$ of the hesitant participants) as the most common reasons behind vaccine hesitancy/rejection [50]. The second also reported that the willingness to get COVID-19 vaccination was positively correlated with the perception of vaccine safety [51]. Another study from Egypt during January-March 2021 with 1,011 participants from the general public reported that COVID-19 vaccine acceptance rate was only $25 \%$ and a majority of participants expressed strong worries about unforeseen effects of the vaccine [49].

Another important result that was consistent with previous studies was the observation of a lower levels of COVID-19 vaccine acceptance rates in countries of the Middle East and North Africa and the generally very high levels of vaccine acceptance in South American countries. An early systematic review on the global rates of COVID-19 vaccine acceptance noticed similar results and the low levels of vaccine acceptance in the Middle East region was attributed to generally high levels of embrace of conspiracy beliefs regarding the origin of SARS-CoV-2 and the COVID-19 vaccines [52-54]. For example a study that was conducted in December 2020 among participants from Arab countries (with a majority from Jordan and Kuwait), reported that about a quarter of the participants believed that COVID-19 vaccines are intended to inject microchips into recipients (27.7\%) and that the vaccines are related to infertility (23.4\%) [26]. This result was also seen among university students in Jordan, with a significant correlation between vaccine conspiracy beliefs and vaccine hesitancy/rejection [53]. Such a low prevalence of vaccine acceptance can also be linked to lower levels of confidence (in governments, 
pharmaceutical companies and healthcare providers), adversely affecting the intentions to get vaccinated [55].

To the contrary, the highest levels of COVID-19 vaccine acceptance rates reported in this study were seen in the two South American countries (Brazil and Chile). A previous study that was conducted in February 2021, which investigated COVID-19 vaccine acceptance in various countries of South America and the Caribbean reported generally high levels of vaccine acceptance, which were in line with our results [56]. Specifically, COVID-19 vaccine acceptance rate in Brazil was reported to be $83 \%$ compared to $95 \%-98 \%$ across the four different scenarios of vaccine safety and efficacy profiles used in this study. In Chile, Urrunaga-Pastor et al, reported a vaccine acceptance rate of $74 \%$, compared to $95 \%$ for scenario of $75 \%$ efficacy with $5 \%$ side effects, and $78 \%$ for the scenario of $50 \%$ efficacy with $5 \%$ side effects [56]. In the low fraction of hesitant individuals in the study by Urrunaga-Pastor et al, fear of side effects was reported as a major contributing factor to lower intentions of COVID-19 vaccination [56].

The strength of the current study can be related to evaluation of the correlation of different scenarios of COVID-19 vaccine safety and efficacy with intentions to get COVID-19 vaccination at a multinational level. Limitations of the current study were inevitable and included low sample size from a few countries (e.g., Egypt); however, the finding of similar trends in COVID19 vaccine acceptance rates compared to larger domestic studies support the findings of this study. Another limitation inherent to online survey studies is related to potential sampling bias towards people who are actively using social media platforms and who had proper internet access.

\section{Conclusion}

The correlation between COVID-19 vaccination intentions with safety and efficacy of vaccines was evident in this study. At the same efficacy level of $75 \%$, the higher possibility of side effects (fever, localized pain) was correlated with a noticeable decline (>20\% drop) in COVID-19 vaccine acceptance rates in the majority of countries in this study. Such a result highlights the potential importance of spreading clear, accurate messages regarding vaccine safety and efficacy, which in turn can help to boost the general public confidence in COVID-19 vaccination. Subsequently, this can help to address the challenges of successful COVID-19 vaccine roll out observed in several LMICs worldwide that was hampered by the phenomenon of COVID-19 vaccine hesitancy and resistance. The lowest rates of COVID-19 vaccine acceptance were observed in the Middle East/North African countries (Egypt, Tunisia, Iran) which requires special attention and well-informed interventional measures to tackle such a widely prevalent problem in the region.

\section{Ethics approval}

This study was approved by the Institutional Review Board of the Universitas Syiah Kuala Zainoel Abidin Hospital and National Health Research and Development Ethics Commission (KEPPKN) of the Ministry of Health of the Republic of Indonesia (\#1171012P).

\section{Acknowledgments}

We are deeply grateful for the support by collators

\section{Conflict of interest}

The authors declare that they have no competing interests.

\section{Funding}

This study was funded by Universitas Syiah Kuala (The Ministry of Education, Culture, Research, and Technology) - H-Index Research Scheme Financial Year 2021 (169/UN11/SPK/ PNBP/2021). 


\section{Underlying data}

Derived data supporting the findings of this study are available from the first author on request.

\section{How to cite}

Rosiello DF, Anwar S, Yufika A, et al. Acceptance of COVID-19 vaccination at different hypothetical efficacy and safety levels in ten countries in Asia, Africa, and South America. Narra J 2021; 1(3): e55 - http://doi.org/10.52225/narra.v1i3.55.

\section{References}

1. Rodriguez-Morales AJ, Cardona-Ospina JA, Gutiérrez-Ocampo E, et al. Clinical, laboratory and imaging features of COVID-19: A systematic review and meta-analysis. Travel medicine and infectious disease 2020; 34:101623.

2. Harapan H, Itoh N, Yufika A, et al. Coronavirus disease 2019 (COVID-19): A literature review. Journal of infection and public health 2020; 13(5):667-673.

3. Farsalinos K, Poulas K, Kouretas D, et al. Improved strategies to counter the COVID-19 pandemic: lockdowns vs. primary and community healthcare, Toxicol. Rep. 8 (2021) 1-9. In.

4. COVID-19 coronavirus pandemic. Available from: https://www.worldometers.info/coronavirus/. Accessed: 1 September 2021.

5. Devi S. COVID-19 resurgence in Iran. Lancet (London, England) 2020; 395(10241):1896.

6. Shimizu K, Wharton G, Sakamoto H, et al. Resurgence of covid-19 in Japan. BMJ 2020;18;370:m3221.

7. Viana J, van Dorp $\mathrm{CH}$, Nunes $\mathrm{A}$, et al. Controlling the pandemic during the SARS-CoV-2 vaccination rollout. Nat Commun 2021; 12(1):3674.

8. Harrison EA, Wu JW. Vaccine confidence in the time of COVID-19. Eur J Epidemiol 2020; 35(4):325-330.

9. Qualls N, Levitt A, Kanade N, et al. Community mitigation guidelines to prevent pandemic influenza-United States, 2017. Morb Mortal Wkly Rep 2017; 66(1):1-34.

10. Lurie N, Saville M, Hatchett R, et al. Developing Covid-19 vaccines at pandemic speed. N Engl J Med 2020; 382(21):1969-1973.

11. Sharpe HR, Gilbride C, Allen E, et al. The early landscape of coronavirus disease 2019 vaccine development in the UK and rest of the world. Immunology 2020; 160(3):223-232.

12. Times TNY. Coronavirus Vaccine Tracker. Available from: https://www.nytimes.com/interactive/2020/science/ coronavirus-vaccine-tracker.html. Accessed: 11 October 2021.

13. WHO Coronavirus (COVID-19) Dashboard. Available from: https://covid19.who.int. Accessed: 4 March 2021

14. Our Word in Data. Coronavirus Pandemic (COVID-19). 2020. Available from: https://ourworldindata.org/covid-cases. Accessed: 11 October 2021.

15. Tagoe ET, Sheikh N, Morton A, et al. COVID-19 vaccination in lower-middle income countries: national stakeholder views on challenges, barriers, and potential solutions. Front Public Health 2021:709127

16. Dhama K, Sharun K, Tiwari R, et al. COVID-19 vaccine hesitancy-reasons and solutions to achieve a successful global vaccination campaign to tackle the ongoing pandemic. Hum Vaccin Immunother 2021;17(10):3495-3499.

17. Lazarus JV, Ratzan SC, Palayew A, et al. A global survey of potential acceptance of a COVID-19 vaccine. Nature Med 2021; 27(2):225-228.

18. Nossier SA. Vaccine hesitancy: the greatest threat to COVID-19 vaccination programs. J Egypt Public Health Assoc 202;96:18

19. Sallam M. COVID-19 Vaccine hesitancy worldwide: a concise systematic review of vaccine acceptance rates. Vaccines 2021; 9(2):160.

20. MacDonald NE. Vaccine hesitancy: definition, scope and determinants. Vaccine 2015; 33(34):4161-4164.

21. Harapan H, Wagner AL, Yufika A, et al. Acceptance of a COVID-19 vaccine in Southeast Asia: a cross-sectional study in Indonesia. Front Public Health. 2020; 8: 381.

22. Al-Mohaithef M, Padhi BK. Determinants of COVID-19 vaccine acceptance in Saudi Arabia: a web-based national survey. J Multidiscip Healthc 2020; 13:1657. 
23. Jennings W, Stoker G, Bunting $\mathrm{H}$, et al. Lack of trust, conspiracy beliefs, and social media use predict COVID-19 vaccine hesitancy. Vaccines 2021; 9(6):593.

24. Paul E, Steptoe A, Fancourt D. Attitudes towards vaccines and intention to vaccinate against COVID-19: Implications for public health communications. Lancet Regional Health-Europe 2021; 1:100012.

25. Machida M, Nakamura I, Kojima T, et al. Acceptance of a COVID-19 Vaccine in Japan during the COVID-19 pandemic. Vaccines 2021; 9(3):210.

26. Sallam M, Dababseh D, Eid H, et al. High rates of COVID-19 vaccine hesitancy and its association with conspiracy beliefs: a study in Jordan and Kuwait among other Arab Countries. Vaccines 2021; 9(1):41.

27. Malik AA, McFadden SM, Elharake J, et al. Determinants of COVID-19 vaccine acceptance in the US. EClinicalMedicine 2020; 26:100495.

28. Fisher KA, Bloomstone SJ, Walder J, et al. Attitudes toward a potential SARS-CoV-2 vaccine: a survey of US adults. Annals of internal medicine 2020; 173(12):964-973.

29. Shekhar R, Sheikh AB, Upadhyay S, et al. COVID-19 vaccine acceptance among health care workers in the United States. Vaccines 2021; 9(2):119.

30. Arce JSS, Warren SS, Meriggi NF, et al. COVID-19 vaccine acceptance and hesitancy in low and middle income countries, and implications for messaging. Nature Med 2021;27:1385-1394.

31. Dyson L, Hill EM, Moore S, et al. Possible future waves of SARS-CoV-2 infection generated by variants of concern with a range of characteristics. Nat Commun 2021; 12(1):5730.

32. Salomoni MG, Di Valerio Z, Gabrielli E, et al. Hesitant or not hesitant? a systematic review on global COVID-19 vaccine acceptance in different populations. Vaccines 2021; 9(8):

33. Wagner A. COVID-19 Vaccine Hesitancy Surveys. In: Inter-university Consortium for Political and Social Research Available from: https://doiorg/103886/E130422V2 2021 (31 August 2021).

34. Akel KB, Masters NB, Shih S-F, et al. Modification of a Vaccine Hesitancy Scale for use in adult vaccinations in the United States and China. Hum Vaccin Immunother 2021;17(8):2639-2646.

35. Billah MA, Miah MM, Khan MN. Reproductive number of coronavirus: A systematic review and meta-analysis based on global level evidence. PLOS ONE 2020; 15(11):e0242128.

36. Anderson RM, Vegvari C, Truscott J, et al. Challenges in creating herd immunity to SARS-CoV-2 infection by mass vaccination. The Lancet 2020; 396(10263):1614-1616.

37. Pormohammad A, Zarei M, Ghorbani S, et al. Efficacy and safety of COVID-19 vaccines: a systematic review and meta-analysis of randomized clinical trials. Vaccines 2021; 9(5):467.

38. Sharif N, Alzahrani KJ, Ahmed SN, et al. Efficacy, Immunogenicity and safety of COVID-19 vaccines: A systematic review and meta-analysis. Front Immunol 2021; 12:7114170.

39. McDonald I, Murray SM, Reynolds CJ, et al. Comparative systematic review and meta-analysis of reactogenicity, immunogenicity and efficacy of vaccines against SARS-CoV-2. npj Vaccines 2021; 6(1):74.

40. James EK, Bokemper SE, Gerber AS, et al. Persuasive messaging to increase COVID-19 vaccine uptake intentions. vaccine 2021; S0264-410X(21)01368-2.

41. Ward JK, Alleaume C, Peretti-Watel P. The French public's attitudes to a future COVID-19 vaccine: The politicization of a public health issue. Soc Sci Med (1982) 2020; 265:113414.

42. Limaye RJ, Sauer M, Truelove SA. Politicizing public health: the powder keg of rushing COVID-19 vaccines. Hum Vaccin Immunother 2021;17(6):1662-1663.

43. Kumar D, Chandra R, Mathur M, et al. Vaccine hesitancy: understanding better to address better. Isr J Health Policy Res. 2016; 5: 2.

44. Baraniuk C. Covid-19: How the UK vaccine rollout delivered success, so far. BMJ 2021; 372:n421.

45. Dagan N, Barda N, Kepten E, et al. BNT162b2 mRNA Covid-19 vaccine in a nationwide mass vaccination setting. N Eng J Med 2021; 384(15):1412-1423.

46. Qunaibi EA, Helmy M, Basheti I, et al. A high rate of COVID-19 vaccine hesitancy in a large-scale survey on Arabs. eLife 2021; 10: e68038

47. Kasrine Al Halabi C, Obeid S, Sacre H, et al. Attitudes of Lebanese adults regarding COVID-19 vaccination. BMC Public Health 2021; 21(1):998.

48. Khaled SM, Petcu C, Bader L, et al. Prevalence and potential determinants of COVID-19 Vaccine hesitancy and resistance in Qatar: results from a nationally representative survey of Qatari nationals and migrants between December 2020 and January 2021. Vaccines 2021; 9(5):471. 
49. Omar DI, Hani BM. Attitudes and intentions towards COVID-19 vaccines and associated factors among Egyptian adults. J Infect Public Health 2021; 14(10):1481-1488

50. Fares S, Elmnyer MM, Mohamed SS, et al. COVID-19 Vaccination perception and attitude among healthcare workers in Egypt. J Prim Care Community Health 2021; 12:21501327211013303.

51. El-Sokkary RH, El Seifi OS, Hassan HM, et al. Predictors of COVID-19 vaccine hesitancy among Egyptian healthcare workers: a cross-sectional study. BMC Infect Dis 2021; 21(1):762.

52. Sallam M, Dababseh D, Yaseen A, et al. COVID-19 misinformation: Mere harmless delusions or much more? $A$ knowledge and attitude cross-sectional study among the general public residing in Jordan. PLOS ONE 2020; 15(12):e0243264.

53. Sallam M, Dababseh D, Eid H, et al. Low COVID-19 vaccine acceptance is correlated with conspiracy beliefs among university students in Jordan. Int J Environ Res Public Health. 2021; 18(5): 2407.

54. Sallam M, Dababseh D, Yaseen A, et al. Conspiracy beliefs are associated with lower knowledge and higher anxiety levels regarding COVID-19 among students at the university of Jordan. Int J Environ Res Public Health 2020; 17(14):4915

55. Solís Arce JS, Warren SS, Meriggi NF, et al. COVID-19 vaccine acceptance and hesitancy in low- and middle-income countries. Nat Med 2021; 27(8):1385-1394.

56. Urrunaga-Pastor D, Bendezu-Quispe G, Herrera-Añazco P, et al. Cross-sectional analysis of COVID-19 vaccine intention, perceptions and hesitancy across Latin America and the Caribbean. Travel Med Infect Dis 2021; 41:102059 